\title{
Numerical modelling of dry stacking tailings heaps
}

\author{
GC Vizcarra Vale S.A., Brazil
}

\begin{abstract}
In the practice of geotechnical engineering applied to mining, dry stacked tailings heap projects are analysed by limit equilibrium methods and stress-strain analyses. These methods and model assumptions do not consider the influence of the partially drained conditions, strain softening, and transition from over-consolidated to normally consolidated behaviour of the foundation. This situation can lead to calculations of deterministic safety factors that are often overestimated, according to the nature of the foundation. This article proposes a methodology for numerical modelling of the foundation during staged construction of a heap, and considers loading time as a critical variable in calculating the Factor of Safety of the heap dry stacked of elaborating a numerical model considering the foundation and construction.
\end{abstract}

The physical and geotechnical properties of the materials were derived through the model, and are the interpretation of data obtained from a geotechnical investigation and laboratory testing campaign. Stress-strain analyses are performed for different stacking speed scenarios to assess its influence on the safety factor at each loading stage. The analyses are coupled, where the distribution of stresses and deformations within the foundation and the dissipation of porewater pressures over time are simulated. The results show the evolution of the Factor of Safety, the spatial distribution of the regions with excess porewater pressure, allowing the optimisation of both the rate of rise of the dry stack and external batter slope for the project to minimise ground improvement.

It is observed that there is a strong dependence of the coefficient of permeability of the foundation soils on the porewater pressure dissipation time. Numerical modelling considering partially drained conditions allows greater understanding of foundation behaviour and performance during the development of the dry stacked heap. The analysis is considered applicable to both soft soil foundation conditions and conversion of existing wet disposal tailings facilities to dry stacking atop, referred to as 'piggybacking' within the mining industry.

Keywords: dry stacking tailings, modelling, partially drained

\section{Introduction}

Each state of Brazil grants a limited available space for the operation of waste disposal facilities to mining companies. One of the most important aspects is the safe and environmentally responsible disposal of mine waste that must meet increasingly stringent environmental standards and regulations for maintaining the stability of the structures. In Brazil, some mining companies have adopted a strategy of substituting wet tailings disposal with the dry stacking of tailings in heaps, to optimise the tailings footprint by allowing to construct higher and to recover more process water. The height of these heaps can vary between 30-250 m, while the heaps can be developed over a period of up to 10 years. The stability of these heaps is analysed mainly using a limit equilibrium approach, which does not consider construction time, nor progressive movements within the change of material characteristics over time.

Moreover, there is a variation in porewater pressure and stresses in the soil mass during the heap construction. This article shows a design approach that illustrates the impact of stress and porewater pressure variations on the Factor of Safety of the heap throughout the construction period. Related studies include Priscu (1999), Zardari (2011), Martin \& McRoberts (1999), and Lara et al. (2013). 


\section{Methodology}

The study includes a 2D stress-strain analysis coupled with a consolidation analysis of a section of a dry stacked tailings heap on a soft soil or soft tailings foundation. The model geometry is based on a typical configuration of a $30 \mathrm{~m}$ high heap and considers a stacking lift height of $2 \mathrm{~m}$. Figure 1 depicts the model geometry analysed as part of this study.

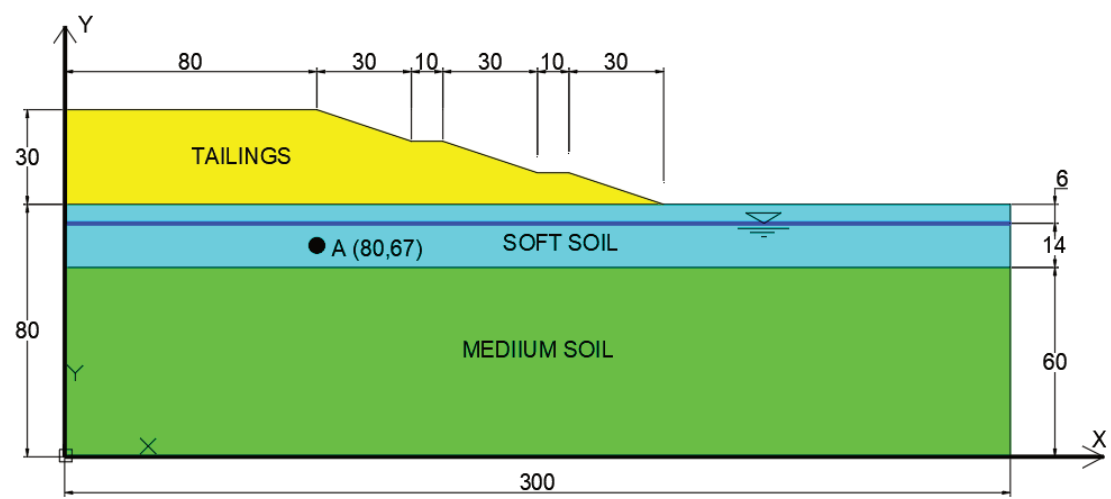

\section{Figure 1 Model geometry}

Table 1 shows the average materials properties across Brazilian sites.

\section{Table 1 Materials properties}

\begin{tabular}{|c|c|c|c|c|}
\hline Properties & Units & Medium soil & Soft soil & Dry stacking tailings \\
\hline Depth & $\mathrm{m}$ & 20 to 80 & 0 to 20 & \\
\hline Unit weight & $\mathrm{kN} / \mathrm{m}^{3}$ & 18 & 16 & 21 \\
\hline Saturated unit weight & $\mathrm{kN} / \mathrm{m}^{3}$ & 18.5 & 16.5 & 21.5 \\
\hline Constitutive model & & Mohr-Coulomb & Soft soil & Soft soil \\
\hline Young modulus, E & $\mathrm{MPa}$ & 60 & & \\
\hline Poisson coefficient, $v$ & & 0.3 & & \\
\hline Compressibility index, Cc & & & 0.3 & 0.1 \\
\hline Swelling index, Cs & & & 0.03 & 0.01 \\
\hline Initial void ratio, $\mathrm{e}_{0}$ & & & 1.2 & 1 \\
\hline Cohesion, c & & 20 & 2 & 2 \\
\hline Friction angle, $\phi$ & & 36 & 32 & 30 \\
\hline Over-consolidation ratio & & & 1 & 1 \\
\hline $\begin{array}{l}\text { Isotropic hydraulic } \\
\text { conductivity, K }\end{array}$ & $\mathrm{m} / \mathrm{d}$ & $\begin{array}{l}1 \\
\left(1.16 \times 10^{-5} \mathrm{~m} / \mathrm{sec}\right)\end{array}$ & & $\begin{array}{l}1 \times 10^{-2} \\
\left(1.16 \times 10^{-7} \mathrm{~m} / \mathrm{sec}\right)\end{array}$ \\
\hline Case K1 & & & $\begin{array}{l}1 \times 10^{-2} \\
\left(1.16 \times 10^{-7} \mathrm{~m} / \mathrm{sec}\right)\end{array}$ & \\
\hline Case K2 & & & $\begin{array}{l}1 \times 10^{-3} \\
\left(1.16 \times 10^{-8} \mathrm{~m} / \mathrm{sec}\right)\end{array}$ & \\
\hline Case K3 & & & $\begin{array}{l}5 \times 10^{-4} \\
\left(5.79 \times 10^{-9} \mathrm{~m} / \mathrm{sec}\right)\end{array}$ & \\
\hline
\end{tabular}


The medium soil has a high permeability that facilitates the water flux. The built-in constitutive model 'soft soil,' implemented in the software Plaxis 8 , was used to model the soft soil foundation and dry stacked tailings layers. The influence of the variation of soft soil foundation permeability is analysed in three scenarios with different permeability coefficients K1, K2, and K3.

Moreover, three scenarios were modelled to evaluate the influence of different tailings stacking rates. Table 2 depicts these scenarios.

\section{Table 2 Stacking rate scenarios}

\begin{tabular}{ll}
\hline Scenario & Stacking rate \\
\hline T1 & $1 \mathrm{~m} /$ month \\
T2 & $2 \mathrm{~m} /$ month \\
T3 & $4 \mathrm{~m} /$ month \\
\hline
\end{tabular}

Plaxis 8 was used to model the section through triangular elements. The initial condition was the ground without the heap, where initial pore pressures and stresses were generated. After that, each $2 \mathrm{~m}$ thickness layer was included and analysed to complete the heap with a height of $30 \mathrm{~m}$.

\section{$3 \quad$ Results}

Figures 2 and 3 show the excess pore pressure distribution immediately and 15 days after construction of the fifteenth layer, respectively. The pore pressure distributions depicted are also based on a soft soil foundation with permeability K2 $=1 \times 10^{-3} \mathrm{~m} /$ day $\left(1.16 \times 10^{-8} \mathrm{~m} / \mathrm{sec}\right)$ and a stacking rate $\mathrm{T} 3=4 \mathrm{~m} / \mathrm{month}$. The foundation was partially drained after 15 days.

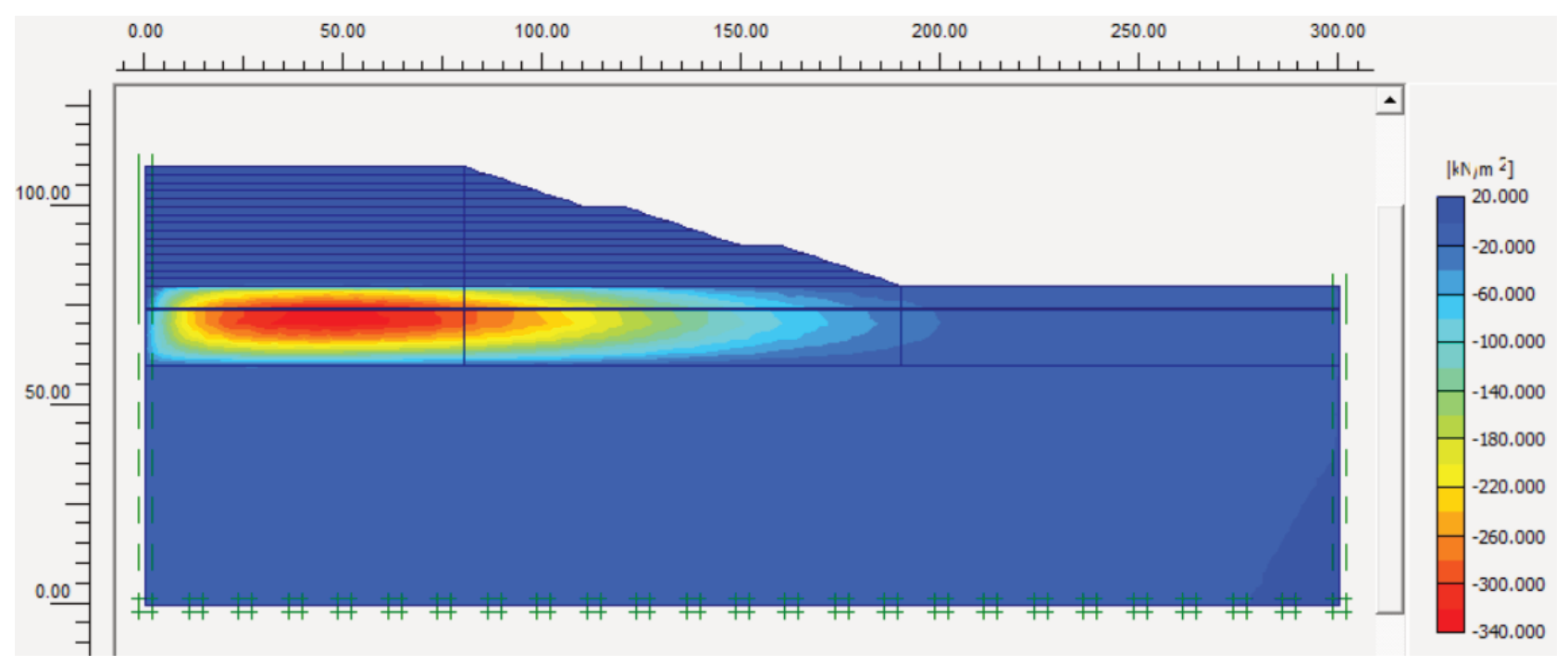

Figure 2 Excess pore pressure distribution after fifteenth layer construction (case K2 T3) 


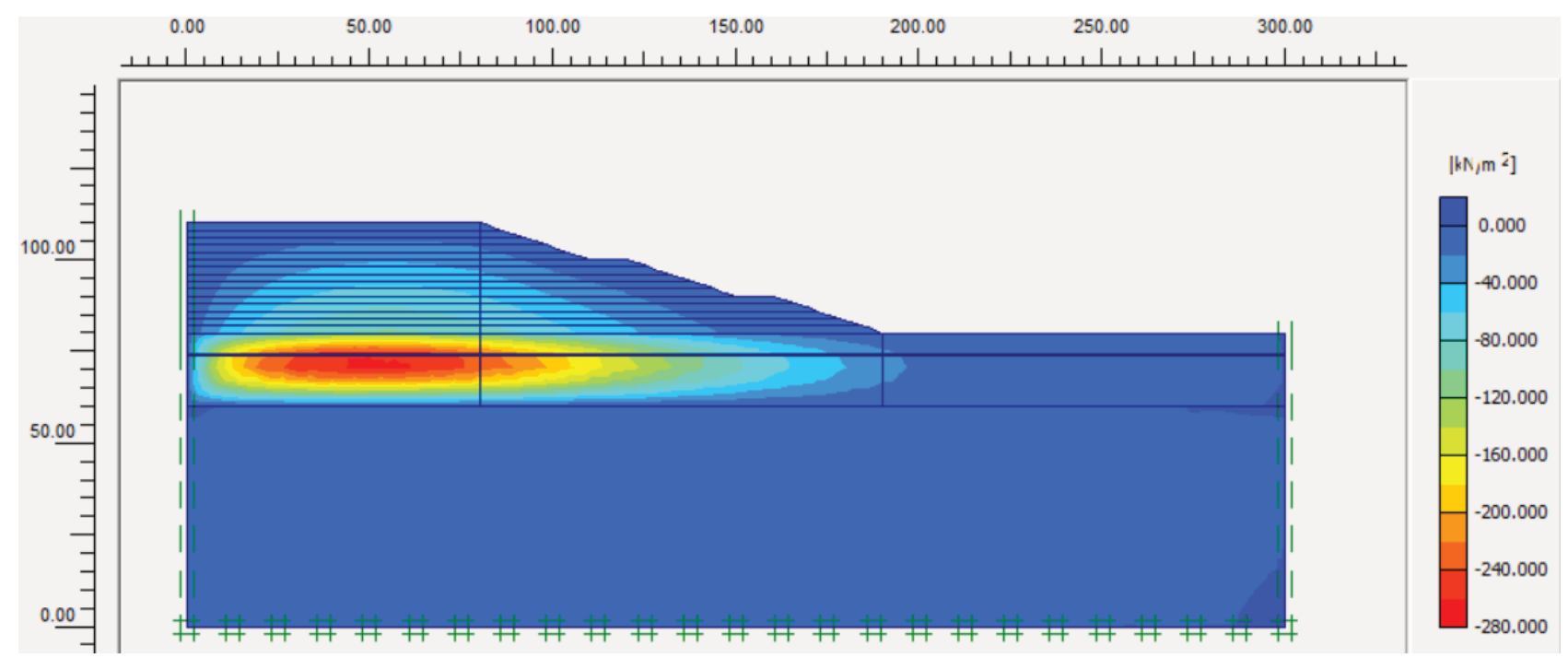

Figure 3 Excess pore pressure distribution at 15 days after fifteenth layer construction (case K2 T3)

Figures 4, 5, and 6 depict excess pore pressure evolution over time for different permeability values and stacking rates. The values correspond to point $A$, as presented in Figure 1.

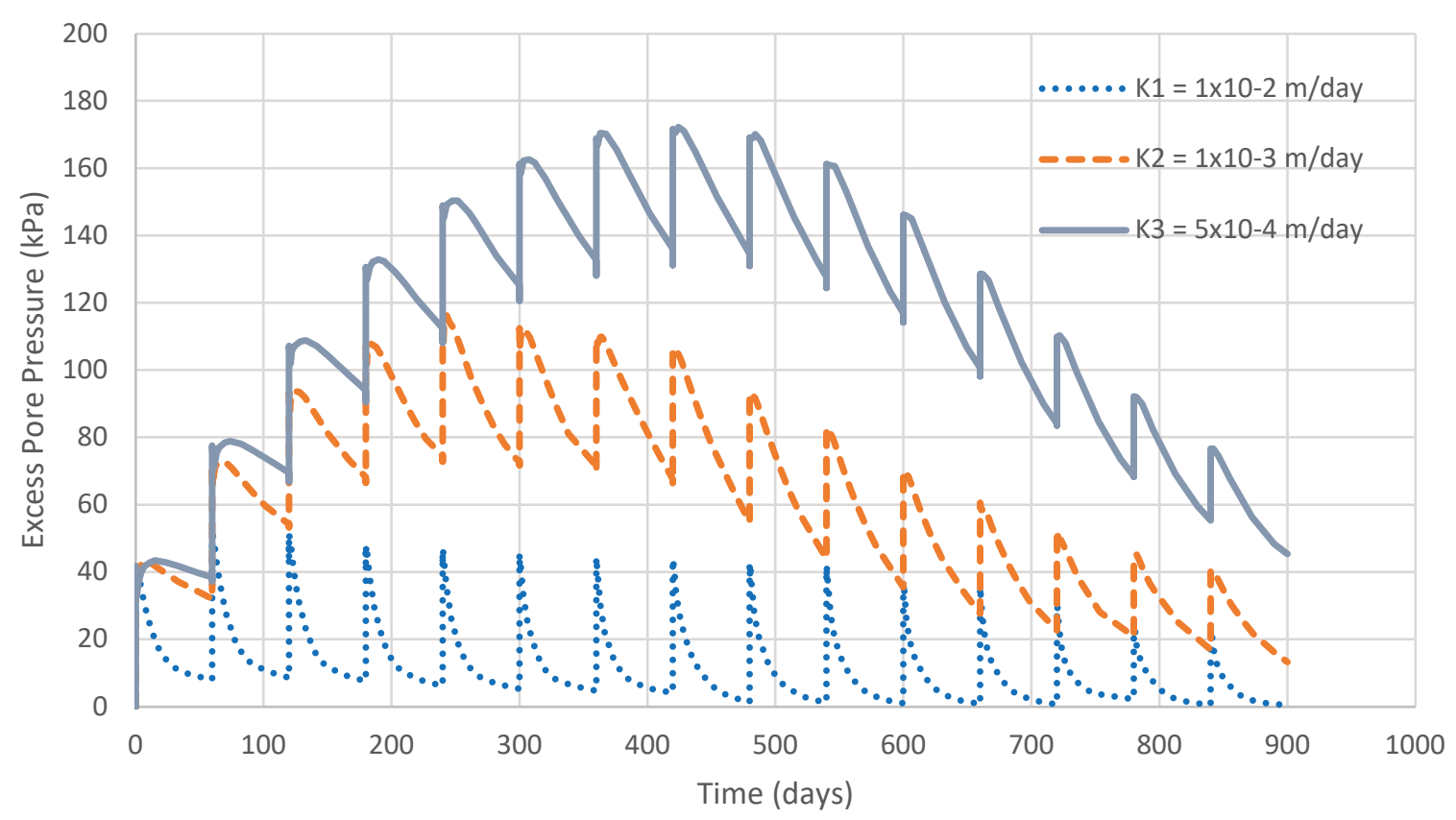

Figure 4 Influence of the soft soil permeability on the excess pore pressure dissipation over time (stacking rate $=1 \mathrm{~m} /$ month) 


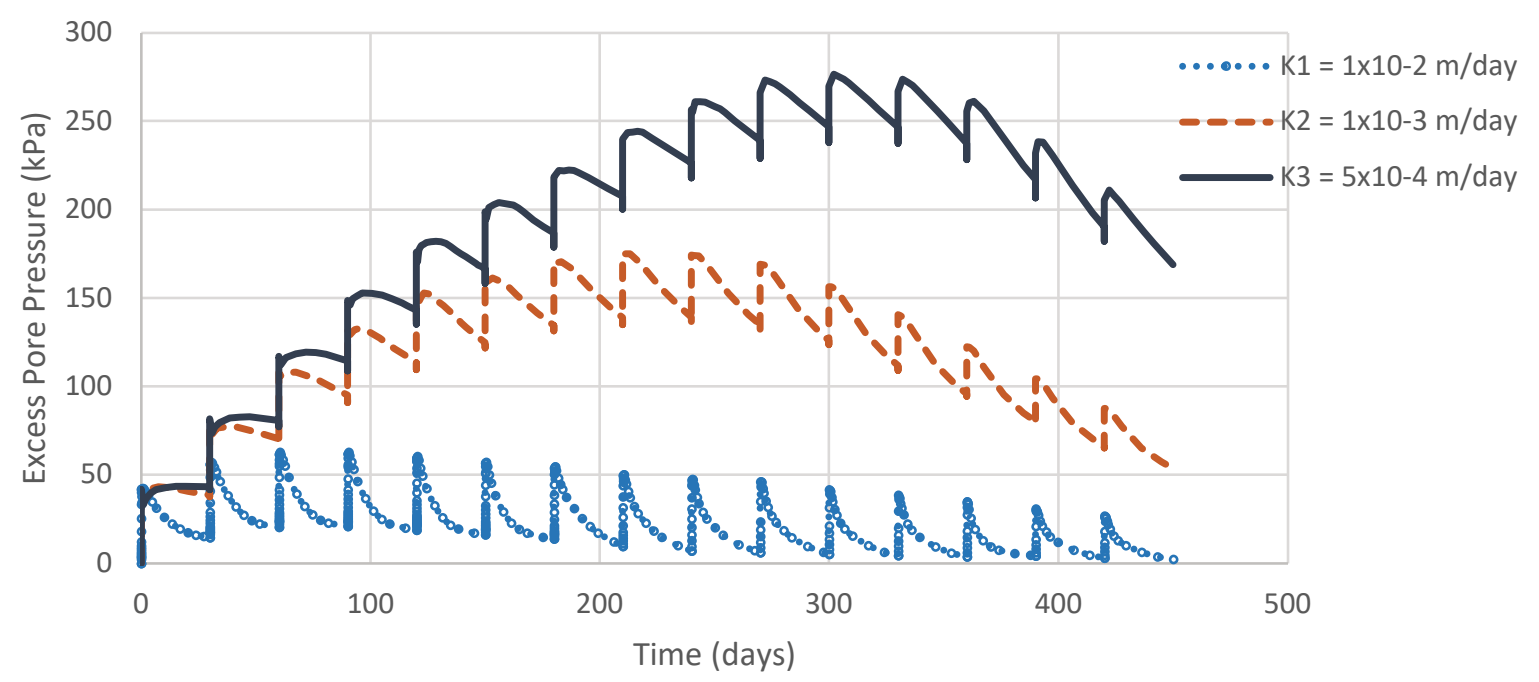

Figure 5 Influence of the soft soil permeability on the excess pore pressure dissipation over time (stacking rate $=2 \mathrm{~m} /$ month)

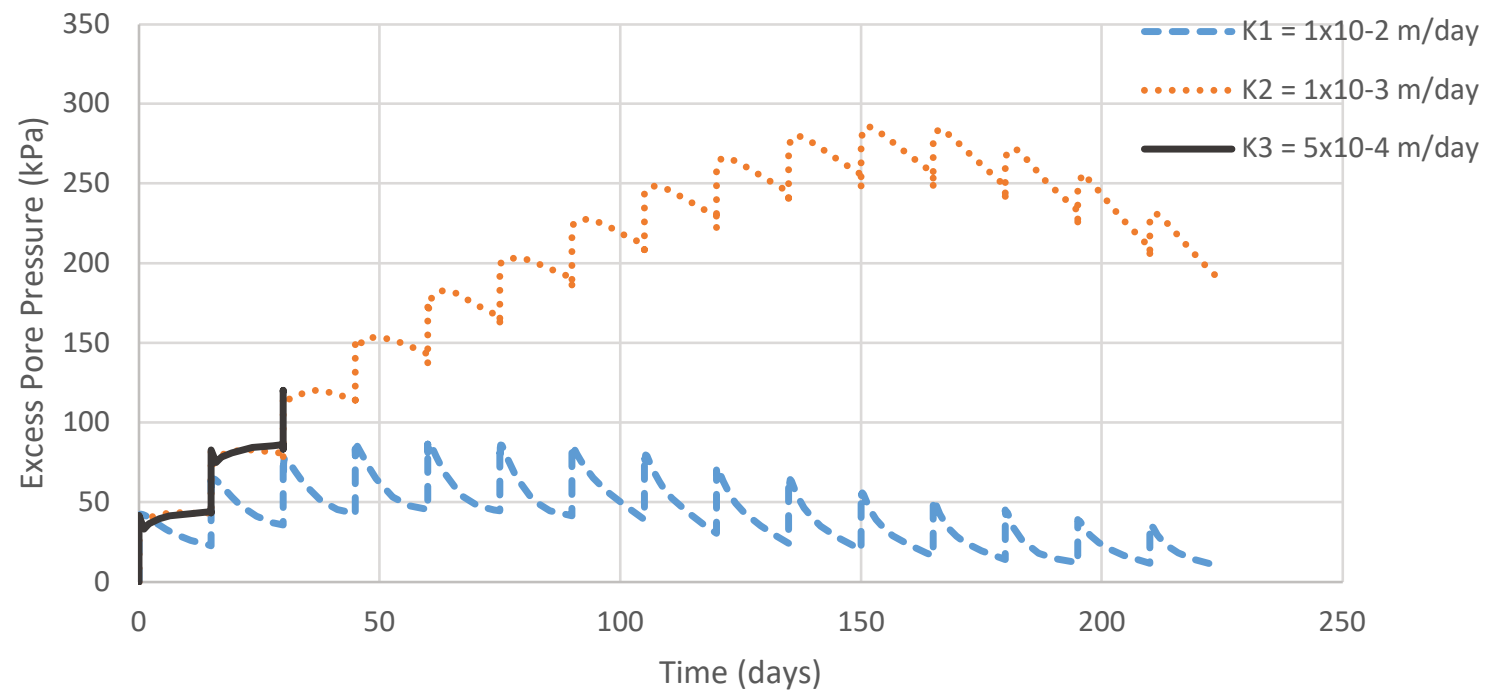

Figure 6 Influence of the soft soil permeability on the excess pore pressure dissipation over time (stacking rate $=4 \mathrm{~m} /$ month)

All scenarios show a strong correlation between permeability, stacking rate, and excess pore pressure dissipation. Low permeability values produce higher pore pressures, which is more significant when the stacking rate is higher. Figure 7 shows the influence of the stacking rate on the pore pressure distribution. A higher stacking rate produces higher pore pressures. In this case, the excess pore pressure is three times higher when the stacking rate is four times faster. 


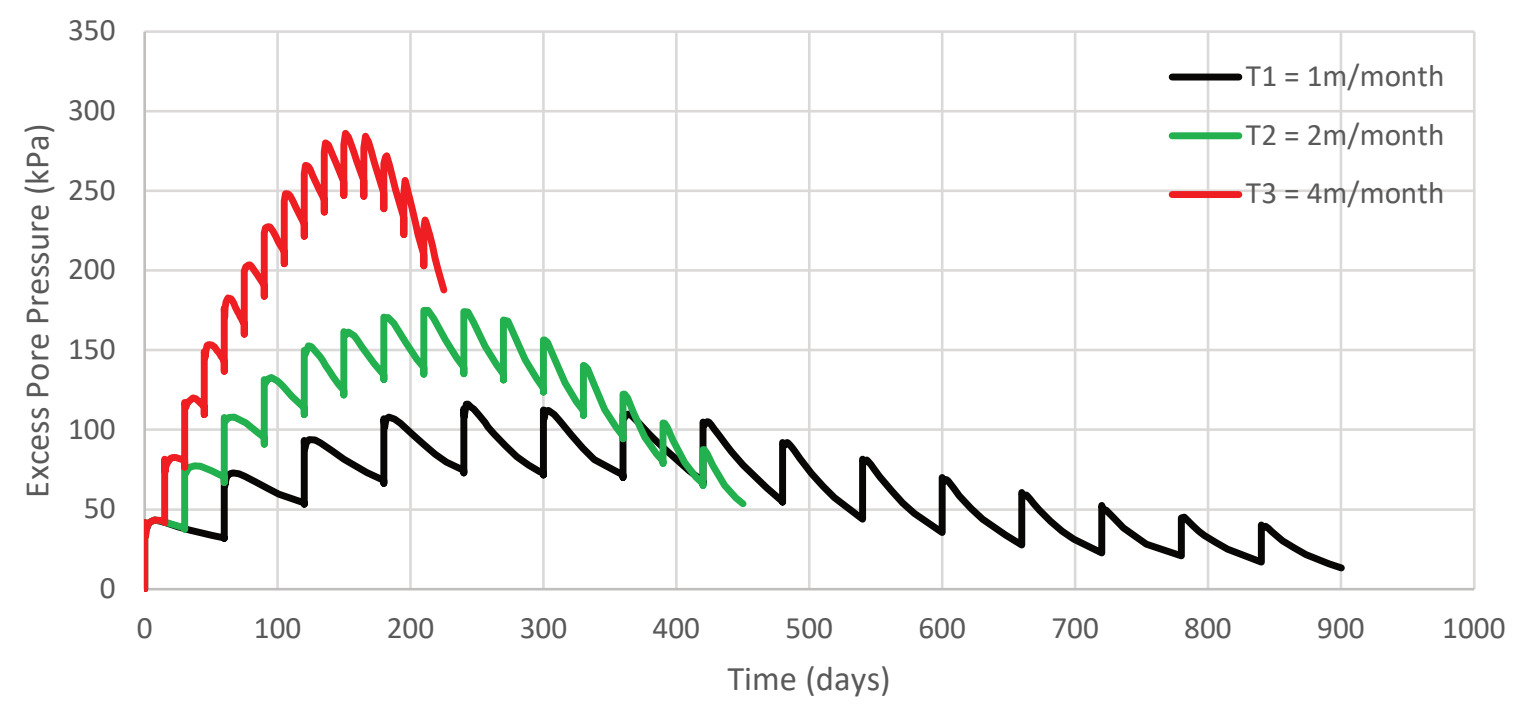

Figure 7 Influence of the stacking rate on pore pressure dissipation (case K2 =1 $\times 10^{-3} \mathrm{~m} /$ day or $1 \times 10^{-8} \mathrm{~m} / \mathrm{s}$ )

Figure 8 shows the influence of stacking rate on the heap Factor of Safety calculated by the strength reduction factor method in Plaxis. For permeability case K1, the stacking rate does not influence the Factor of Safety. For permeability cases $\mathrm{K} 2$ and $\mathrm{K} 3$, it is observed that the factors of safety reduce as the stacking rate increases. For the scenario with the lowest permeability case (K3), the heap collapses when the third layer is constructed.

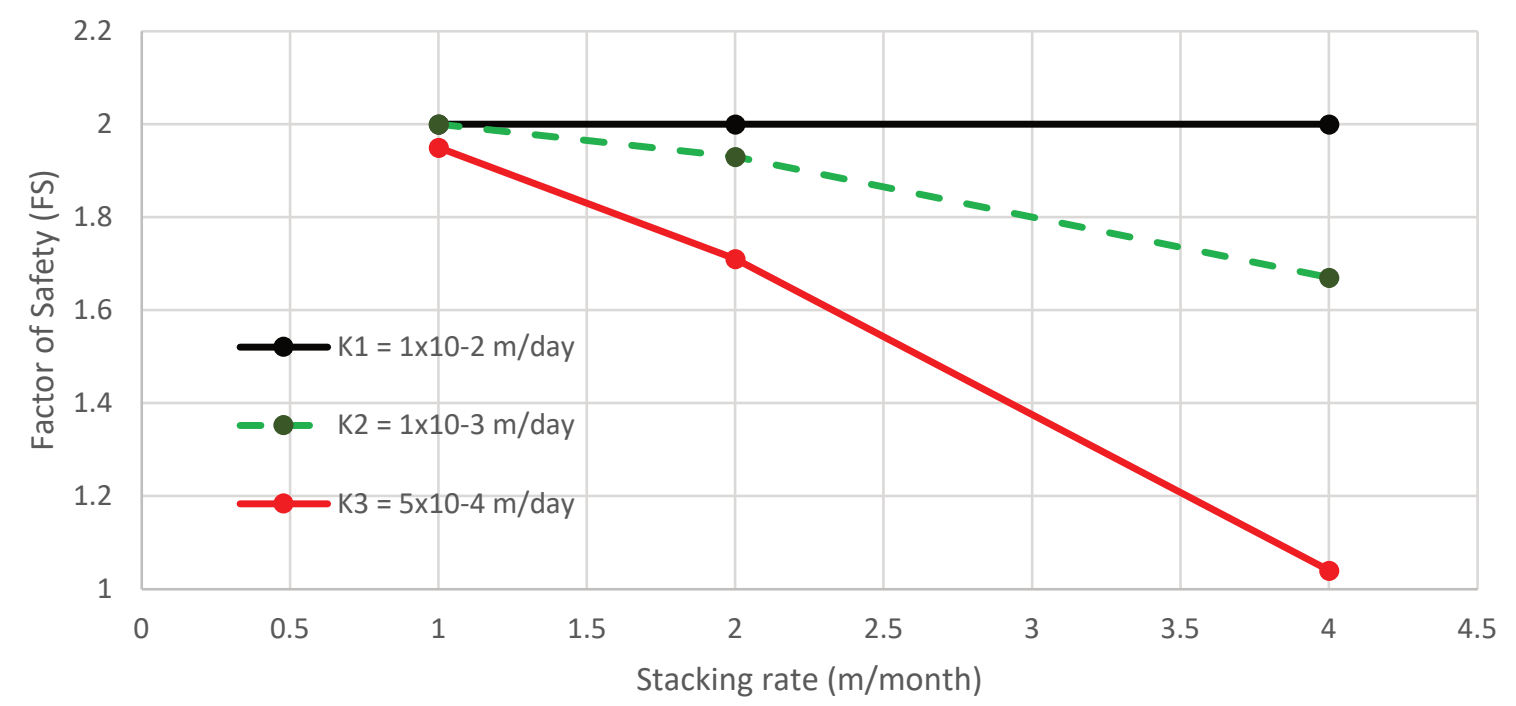

Figure 8 Influence of stacking rate on the heap Factor of Safety

\section{Conclusion}

The numerical modelling indicates a strong relationship between foundation permeability, tailings stacking rate, and the rate of pore pressure dissipation. As the permeability reduces, excess pore pressures dissipate slowly under the increased stacking rates, negatively impacting the stability of the tailings heap. Essentially, the lower permeability induces a higher pore pressure which results in provoking a reduction in the Factor of Safety with increasing rate of rise, leading to collapsing.

The values obtained in this paper are valid just for the case analysed, but this procedure can be used to evaluate a safe stacking rate based on specific site conditions. 
Key to understanding the stability of the stack at the design stage are the geotechnical properties of the stacked tailings and the foundation tailings/subgrade. These are primarily the permeability, drained/ undrained strengths, anisotropy and brittleness under Ko conditions. The choice of numerical modelling software (FLAC or Plaxis) will then be dictated by the complexity of the soil behaviour to be modelled.

Where the modelled rate of rise leads to unacceptably low Factors of Safety, the design may need to include additional measures to maintain stability, such as hold points for excess pore pressures to dissipate, an underdrainage system or reduced batter slopes.

Piezometer trigger values can also be developed, from the modelling, to allow the use of the observational approach during heap construction.

\section{References}

Lara, JL, Pornillos, EU \& Muñoz, HE 2013, 'Geotechnical-geochemical and operational considerations for the application of dry stacking tailings deposits - state-of-the-art', in R Jewell, AB Fourie, J Caldwell \& J Pimenta (eds), Paste 2013: Proceedings of the 16th International Seminar on Paste and Thickened Tailings, Australian Centre for Geomechanics, Perth, pp. 249-260, https://doi.org/10.36487/ACG_rep/1363_19_Munoz

Martin, T \& McRoberts, E 1999, 'Some considerations in the stability analysis of upstream tailings dams', Proceedings of the Sixth International Conference on Tailings and Mine Waste '99, Taylor \& Francis, London, pp. 287-302.

Priscu, C 1999, Behavior of Mine Tailings Dams Under High Tailings Deposition Rates, PhD thesis, McGill University, Montreal.

Zardari, M 2011, 'Stability of tailings dams', licentiate thesis, Luleå University of Technology, Luleå. 
